\title{
OBSERVED ANGULAR MOTIONS IN PLANETARY NEBULAE
}

\author{
Martha H. LiLler and William Liller \\ (Harvard College Observatory, Cambridge, Mass., U.S.A.)
}

\begin{abstract}
A BSTRACT
Plates of planetary nebulae taken in October 1965 and June 1967 with the 100-inch and 60-inch telescopes on Mount Wilson are compared with photographs taken with the same instruments 40 or more years previously, to determine the amount of angular motion of material outward from the central stars. Densitometer tracings of the nebulae yield values of $\dot{\theta}$, the radial motion in seconds of arc per century for condensations (filaments, rings, etc.) as well as for the edges of the visible material. Preliminary data are presented for NGC 2392, 6818 and 7662. For NGC 2392 and NGC 7662 distances are derived.
\end{abstract}

\section{Introduction}

Several papers reporting measurements of the angular motion of material outwards from central stars of planetary nebulae have appeared in the past few years (Latypov, 1957; Cudovičeva, 1964; W. Liller, 1965; Liller et al., 1966). The incomplete and, in some cases, conflicting results given in these papers suggest that additional measurements should be made on sets of plates with as large a scale as possible.

With this in mind, one of us (W.L.), in October 1965 and again in June 1967, secured a number of second-epoch plates of planetary nebulae with the 100 -inch and 60 -inch reflectors at Mount Wilson. These plates are being compared with first-epoch plates taken with the same instruments from 1909 to 1925.

Although the reduction of the plate material is incomplete, we can present some preliminary results of our investigation at this time.

\section{Analysis of the Plates}

Using a Baird microdensitometer with a precision drive, we made traces along from 1 to 5 axes of each image of the planetary nebulae. The axes were chosen to pass through the well-defined filaments of the nebula and also to give as even a coverage of position angle as possible. Projected slit sizes, the same for old and new plates taken with a given telescope and focus, ranged from 0.02 to $0.05 \mathrm{~mm}$ in width and 0.15 to $0.6 \mathrm{~mm}$ in length, depending upon the size and detail of the nebula and the plate scale.

To check for any systematic change in plate scale that may have occurred between the early- and late-epoch plates, we measured radial distances of about 6 stars from 
the central stars of 6 nebulae. In all cases average changes in distance amounted to less than a few microns, or less than a few hundredths of a second of arc, at the mean angular distance of the stars, and hence probably less than this amount at the distance of the outer portions of the nebula.

The densitometer tracings were measured in two ways. The first, and most satisfactory procedure when it was possible, was simply to measure the distance from the central star to the position of peak intensity of a filament or portion of a ring, or the distance from one filament peak to another diametrically opposed, if the central star was not detectable. The second procedure was to measure the distance from the central star to the 'edge' of the nebula, where edge is defined to be that point on the tracing at which a straight line, placed through the steeply decreasing trace in the outer region of the nebula, intersects the density of the sky background. The edge measures are less satisfactory than the filament measures because the edge measures are more strongly affected by seeing and because they are dependent on density or length of exposure.

The edge measures can be corrected for density and seeing effects as follows. A comparison star of suitable density is traced on all plates of a nebula in all directions in which the nebula itself was traced. Distance from edge to central star or from edge to edge for the nebula is plotted against the height of the comparison star above the sky background on the trace made for each trace direction and image. Straight-line least-squares solutions with the same slope for both epochs are fitted to the data for each epoch, and the difference in intercept gives the change in size of the nebula over the period in question. The same procedure is followed for the edge-to-center or edge-to-edge distances of the comparison star itself, and the change in size is subtracted from that for the nebula, as a rough correction for seeing and background effects.

\section{The Results}

Preliminary values for centennial radial angular velocity $\dot{\theta}$ (seconds of arc per 100 years) for three of the nebulae are presented below. In the case of the edges of the nebulae, $\dot{\theta}$ may refer either to true motion of the nebular material or change in radius of an ionization sphere. For the ring and filament measures, it is probable that we are measuring motion of the material. Therefore, we compare $\dot{\theta}$ from ring and filament measures with the spectroscopically determined $\Delta V$ of Wilson (1950) to derive the distances to the nebulae. We discuss each nebula in turn.

(1) NGC 2392: Figure 1a is a graph of $\dot{\theta}$ against the angular radius $\theta$ in seconds of arc for this planetary. Data from all position angles are presented. The discrepant point marked with an asterisk refers to a condensation at P.A. $283^{\circ}$ that lies outside the main ring but inside the condensations marking the outer ring or 'fur', and apparently does not share in the motion shown by the rest of the nebular material. This deviating condensation is shown on Figure 2, a photograph of NGC 2392. A least-squares solution through the rest of the points in Figure la gives a slope of 


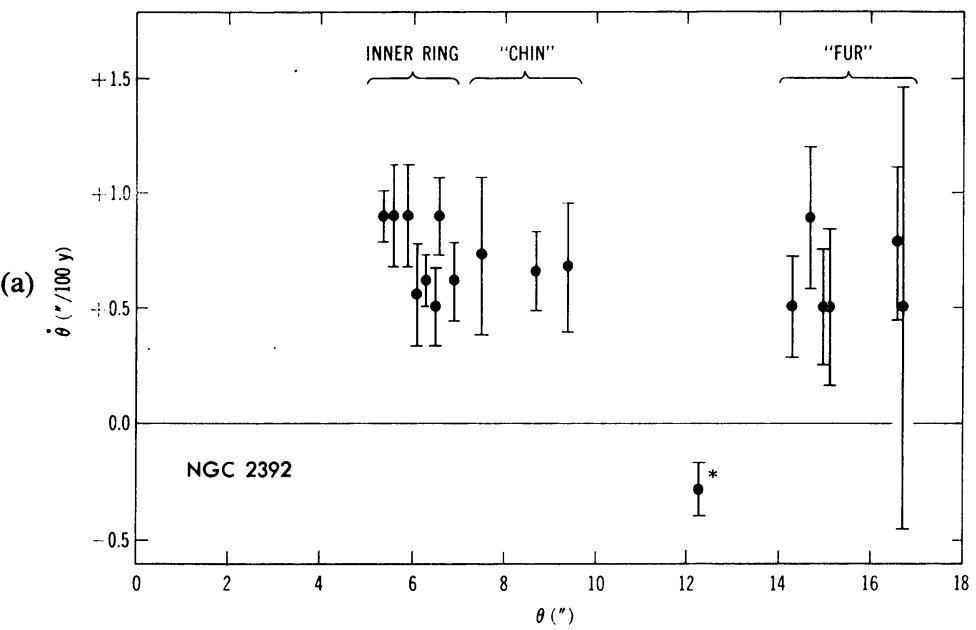

(b)
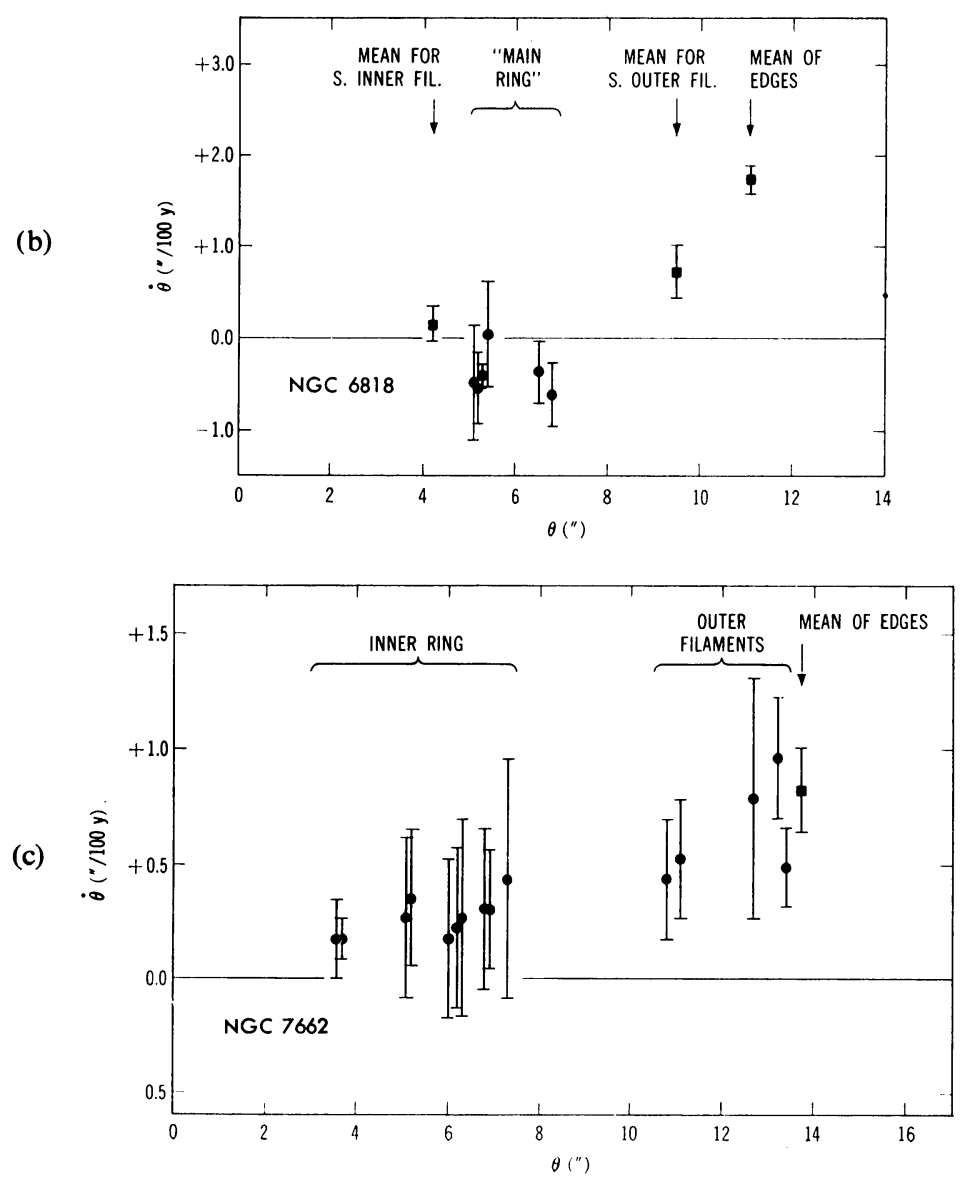


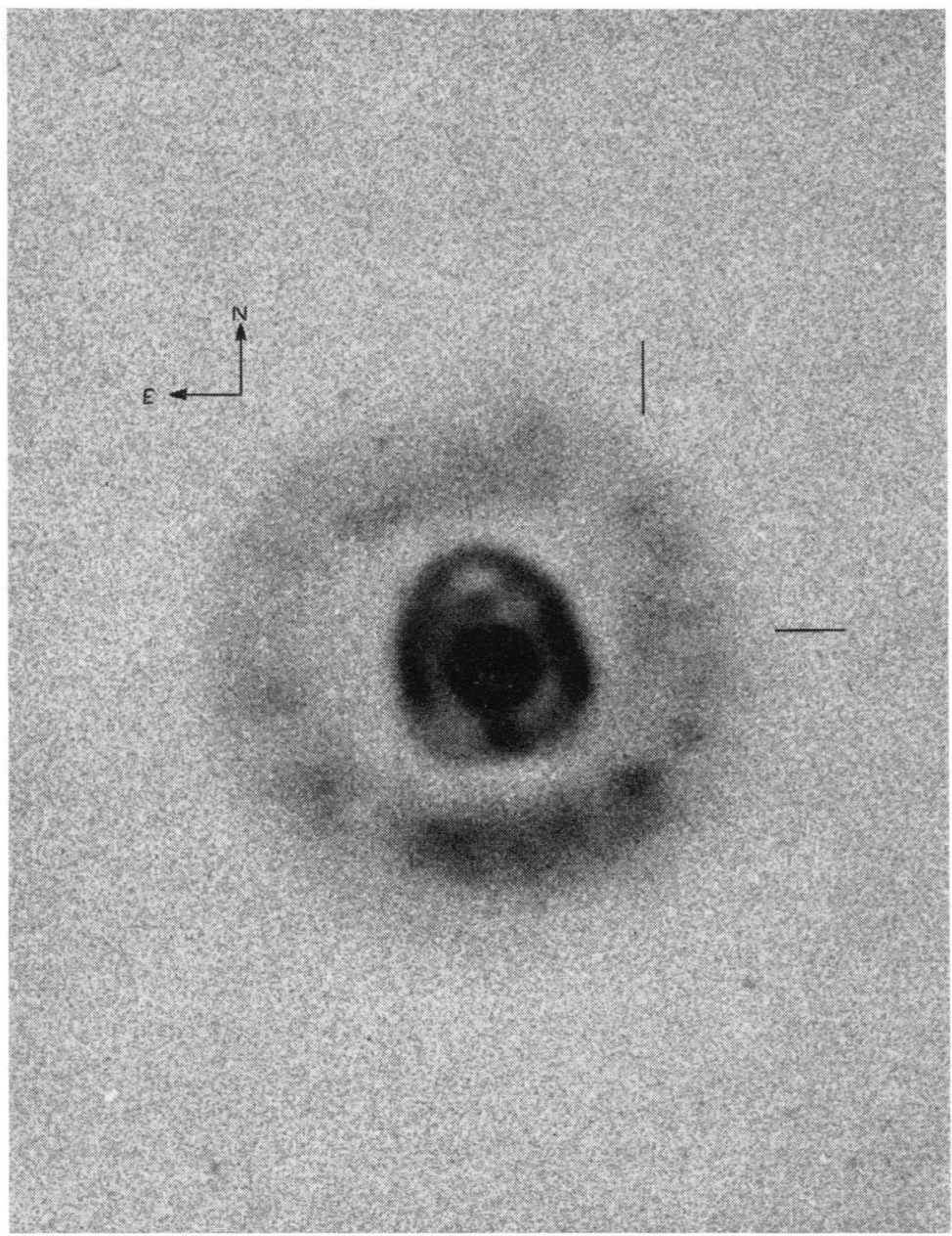

FIG. 2. NGC 2392. The condensation producing the discrepant point in Figure la is marked. Mount Wilson 60-inch Cassegrain photograph.

$-.018 \pm .012^{\prime \prime}$ of arc per 100 years per second of arc; that is, the angular outward velocity decreases slightly as distance from the central star increases. Wilson's $\Delta V$ values, with the exception of those derived from [Nev], are reasonably constant. From the widths of emission lines in the central star spectrum, he concludes that material is being emitted from the star at nearly this same velocity.

Fig. 1a, b, c. Radial expansion in seconds of arc per 100 years as a function of radius for three planetary nebulae. The error bars are twice the mean error in length. The point marked by an asterisk for NGC 2392 is discussed in the text. Data for NGC 2392 and NGC 6818 from 60-inch Cassegrain plates; data for NGC 7662 from 100-inch Cassegrain plates. 
If the inner ring of NGC 2392 is a projection of a spherical shell and outward velocity is constant with radius within that shell, the observed $\dot{\theta}$ will be somewhat smaller than the true one. The difference depends upon the thickness of the shell, and amounts to about $10 \%$ of $\dot{\theta}$ for a shell that has a thickness of one quarter of its radius. In the case of NGC 2392, in which the shell appears quite thin, the error is probably somewhat smaller than this value. If, however, the inner ring is a true ring, then the observed and true $\dot{\theta}$ will be identical. We combine Wilson's $\Delta V / 2=54 \cdot 8 \mathrm{~km} / \mathrm{sec}$ with our mean $\dot{\theta}$ for the inner regions, $+0.72 \pm \cdot 06^{\prime \prime}$ of arc per century, to get a distance of $1600 \pm 130$ parsec. This distance is larger than the distance derived by most other observers, but is close to Seaton's (1966) distance of 1820 parsec.

(2) NGC 6818: Figure $1 \mathrm{~b}$ shows the $\dot{\theta}$ versus $\theta$ relationship for this planetary with, again, data from five diameters presented. The mean points for the South filaments in Figure $1 \mathrm{~b}$ refer to the section of the nebula where a doubling in the ring occurs, and are means of two measures each. Individual values for $\dot{\theta}$ are shown for the East and West portions, or the 'main' ring. Whereas the Southern filaments and the edges demonstrate a $\dot{\theta}$ that increases from zero with increasing $\theta$, the main ring appears to exhibit a negative $\dot{\theta}$ or possible influx of material toward the central star. Wilson's $\Delta V$ measurements increase with increasing image size in somewhat the way our $\dot{\theta}$ measurements for the Southern filaments do. But with the negative $\dot{\theta}$ for the large part of the ring, it is difficult to say which of our measures correspond to his radial velocities, and thus distance is not derived here.

(3) $N G C$ 7662: Figure 1c presents the $\dot{\theta}$ versus $\theta$ data for this nebula. Again, points from 5 diameters are shown. Although NGC 7662 is similar to NGC 2392 in appearance, its $\dot{\theta}(\theta)$ behaves in quite a different way. A least-squares solution through the ring and filament points yields a $\mathrm{d} \dot{\theta} / \mathrm{d} \theta$ of $+\cdot 047 \pm \cdot 007^{\prime \prime}$ of arc per 100 years per second of arc. This solution passes nearly through the origin. Wilson's $\Delta V$ measurements, in a similar fashion, increase with image size for the inner ring, again indicating that the velocity of the particles increases with increasing distance from the nucleus. Since our plates are sensitive primarily to the radiations of [OIII], [NeIII], and $\mathrm{H}$, we adopt Wilson's mean $\Delta V / 2$ for these of $26.0 \mathrm{~km} / \mathrm{sec}$. We assign this linear velocity to our $\dot{\theta},+0 \cdot 26 \pm \cdot 09^{\prime \prime}$ of arc per century at the average radius of the ring, $5 " 7$, to compute a distance of $2100 \pm 730$ parsec for NGC 7662 . This value is again larger than that derived by most observers, but smaller than Seaton's distance of 2500 parsec.

The results given above are preliminary. The final results will be published when reduction of the plates is complete.

\section{Acknowledgment}

It is a pleasure to thank the National Science Foundation for their support of this research. 


\section{References}

Čudovičeva, O.N. (1964) Izv. glav. astr. Obs. Pulkove, 23, 154.

Latypov, A. A. (1957) Pub. astr. Obs. Tashkent (2), 5, 31.

Liller, W. (1965) Publ. astr. Soc. Pacific, 77, 25.

Liller, M.H., Welther, B.L., Liller, W. (1966) Astrophys. J., 144, 280.

Seaton, M.J. (1966) Mon. Not. R. astr. Soc., 132, 113.

Wilson, O.C. (1950) Astrophys. J., 111, 279.

\section{DISCUSSION}

Münch: I would like to make a remark concerning a possible systematic error in the measurement of nebular images arising from the different colour sensitivities of first- and second-epoch plates. The old Mt. Wilson plates, probably Seed 23, have a sensitivity extending into the green-yellow less than that of orthochromatic plates used at present. Consequently the relative contribution of $\mathrm{NI}_{\mathrm{I}}$ and $\mathrm{N}_{\mathrm{II}}$ and $\mathrm{H} \beta$ may be different in two sets of plates. I have encountered this difficulty while measuring expansion rates of novae envelopes.

M. Liller: In addition, the change from silver to aluminum mirrors has increased the importance of [OH] in the newer plates. However, since in many cases individual knots and filaments were measured, the colour response should not be too critical.

$O$ 'Dell: How were the probable errors derived? Since some points fell into the negative expansion (i.e. contraction) region of your plots, can you say with certainty that such contraction occurs?

M. Liller: The probable errors were derived from internal consistency. Most measurements covered at least 3 images. However, we are not yet certain that the measured contraction is real.

Mathews: I would like to ask if the velocities of the outer blobs in NGC 7009, which are moving outward faster than the main nebula, might indicate that these blobs were ejected at a later time.

W. Liller: The outer blobs of NGC 7009 share in a quite well-defined linear relationship between angular velocity and distance from central star. Therefore, it appears that all visible mass left the star at approximately the same instant. 